\title{
Formulation and Validation of Social Cognition Training Program and its Effectiveness on Symptoms of Oppositional Defiant in 7 to 9 Years Old Children
}

Shima Pajouhinia, Hossein Eskandari", Ahmad Borjali, Ali Delavar, Abdollah Moatamedy

Faculty of Psychology and Educational Sciences, Allameh Tabataba'i University, Tehran, Iran

\section{Article Info:}

\section{A BSTRACT}

Introduction: oppositional defiant disorder (ODD) is one of the most common externalizing disorders in childhood and one of the most common psychiatric disorders among clients of health centers. The purpose of this study was to formulate and validate a social cognition training program and determine its effectiveness on symptoms of oppositional defiant. Materials and Methods: The present study was conducted in two stages. In the first stage, after reviewing the literature, therapeutic theories and concepts in the field of social cognition and training program were developed. Then, ten clinical and child psychologists determined the content validity of the training program. In the second stage, a semi-experimental design with pre-test, post-testand follow-up with a control group was used. The statistical population consisted of all male students aged 7 to 9 years in Tehran schools during 2019-2020 that were evaluated by two-stage sampling. In the first stage, screening was performed. In the second stage, 30 children according to inclusion and exclusion criteria were selected and randomly assigned to the experimental and control groups. The social cognition training program was administered to the experimental group for 10 sessions, and after one month, the experimental group was followed up. Data was collected using Hormerson et al. oppositional defiant disorder rating scale. Results: The results indicated that social cognition training program has acceptable content validity. Furthermore, the results of training intervention showed that social cognition training significantly reduced the symptoms of ODD in children and this effect was significant in the follow-up phase. Conclusion: Social cognition training program can be considered as a valid and effective program for alleviation of ODD symptoms in children.

Key words:

1. Child

2. Mental Disorders

3. Therapeutics

*Corresponding Author: Hossein Eskandari

E-mail: sknd@atu.ac.ir 
تدوين و اعتباريابى برنامه آموزش شناخت اجتماعى و تعيين اثربخشى آن بر نشانههاى نافرمانى

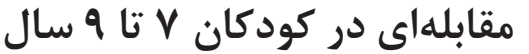

شيما يزوهىنيا، حسين اسكندرى"، احمد برجعلى، على دلاور، عبدالله معتمدى

دانشكده روانشناسى و علوم تربيتى،دانشكاه علامه طباطبائى، تهران، ايران

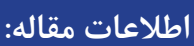

كليد وازهها:

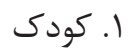

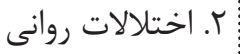

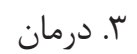

\section{جִ}

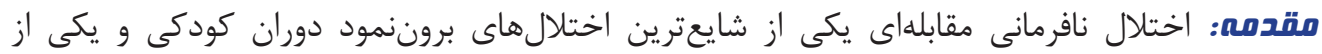

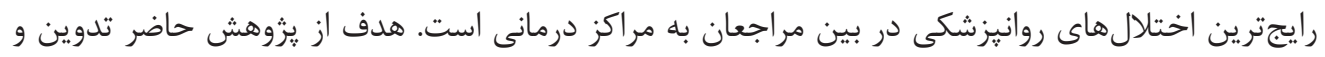

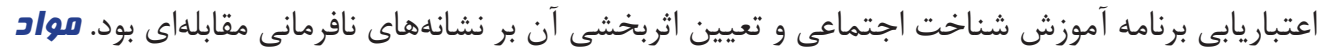

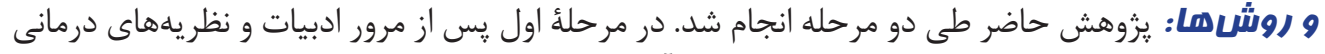

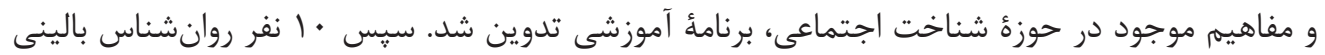

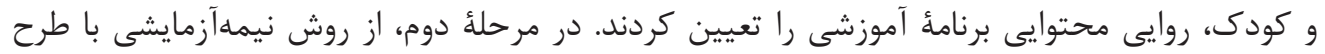

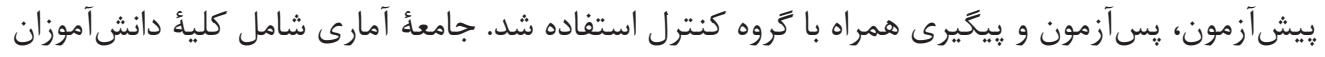

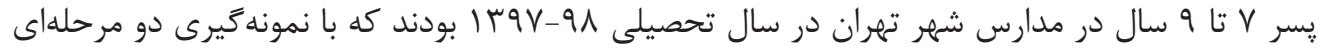

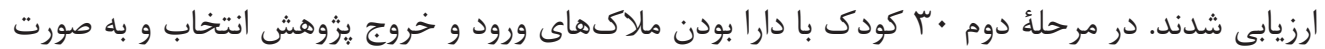

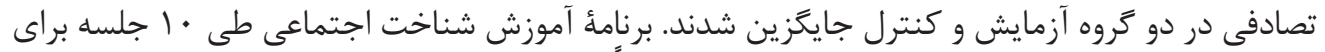

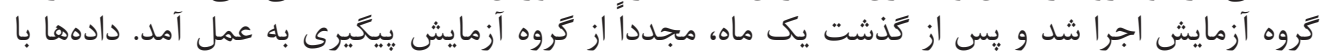

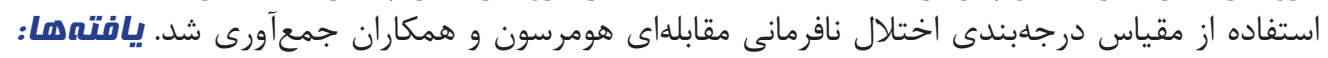

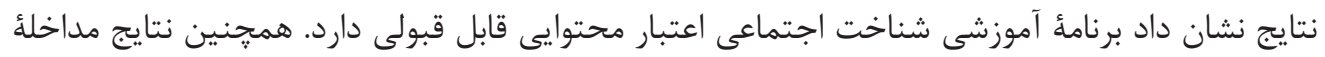

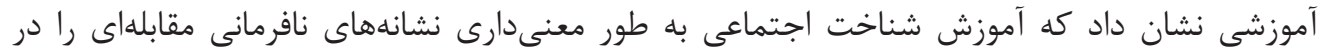

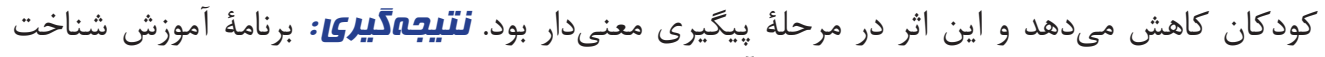

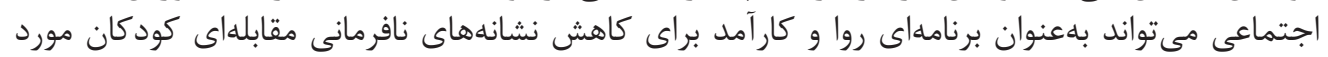
توجه قرار گيرد. 


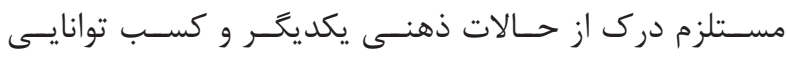

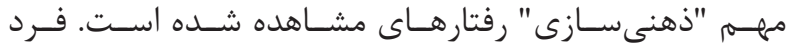

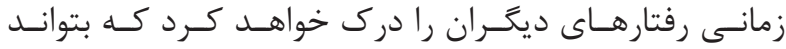

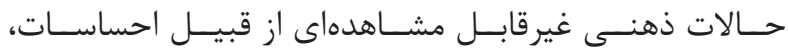

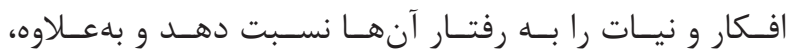

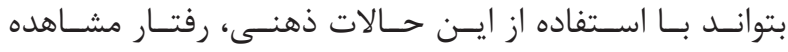

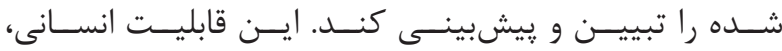

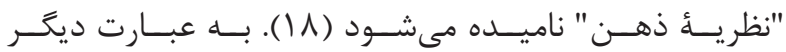

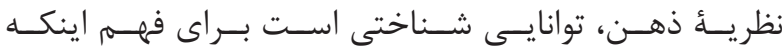

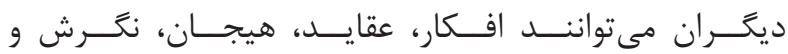

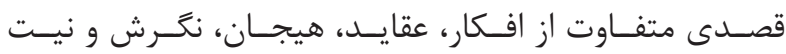

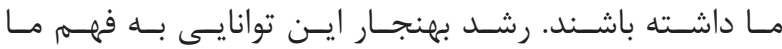

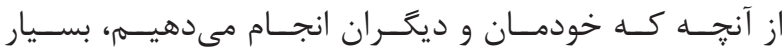

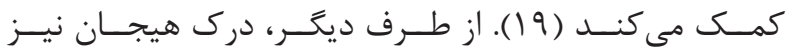

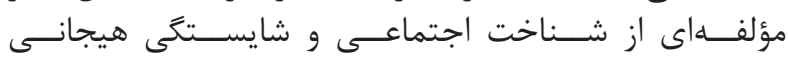

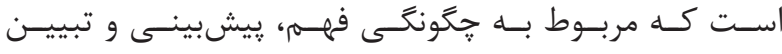

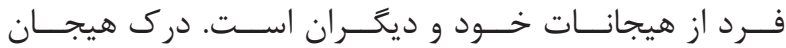

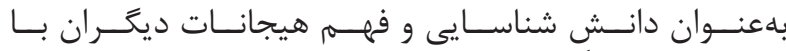

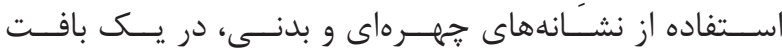

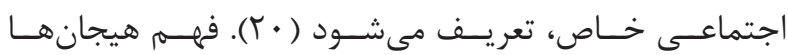

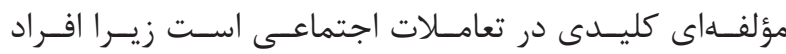

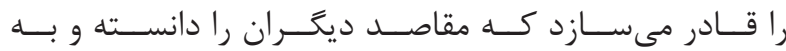

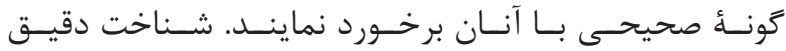

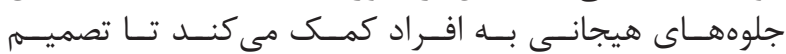

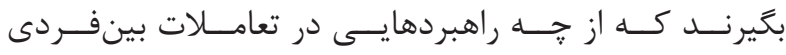

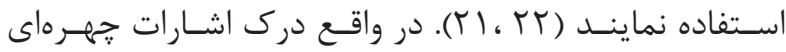

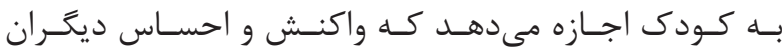

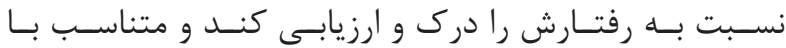

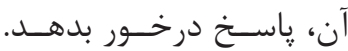

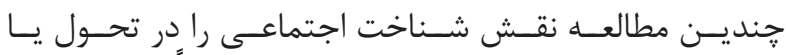

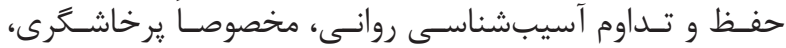

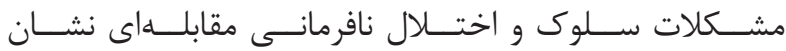

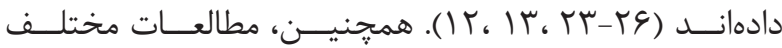

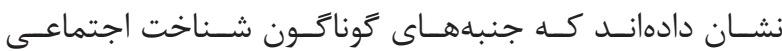

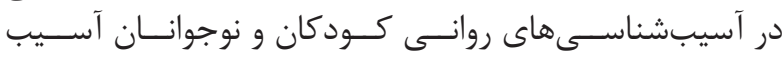

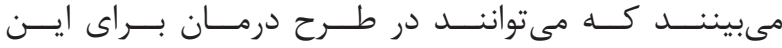

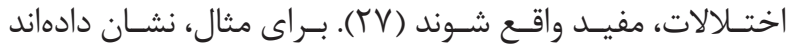

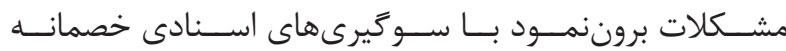

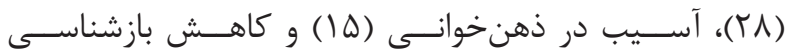

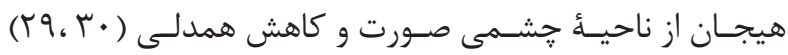

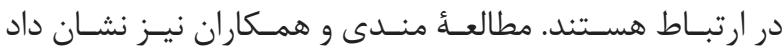

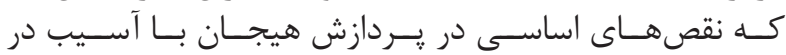

${ }^{1}$ Oppositional defiant disorder

${ }^{2}$ Externalizing

${ }^{3}$ Diagnostic and statistical manual of mental disorders, fifth edition

${ }^{4}$ Angry/irritable mood

${ }^{5}$ Argumentative/defiant behavior

${ }^{6}$ Vindictiveness

${ }^{7}$ Social cognition

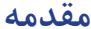

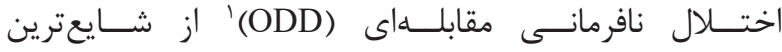

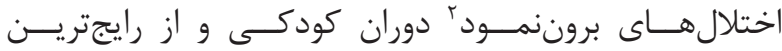

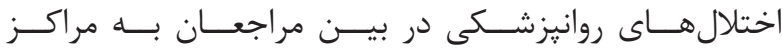

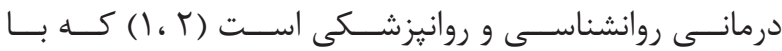

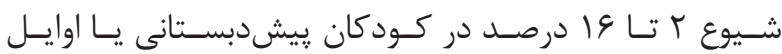

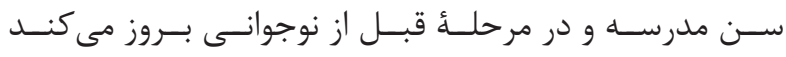

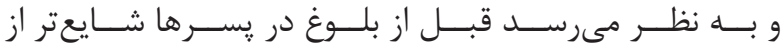

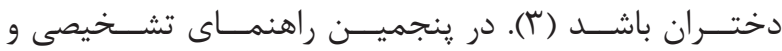

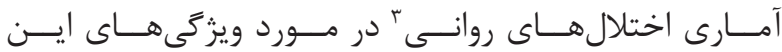

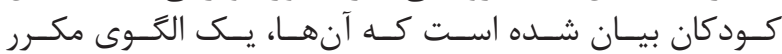

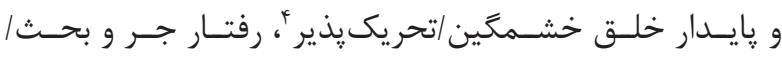

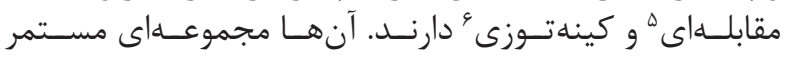

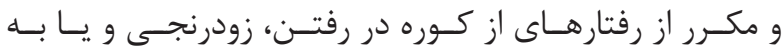

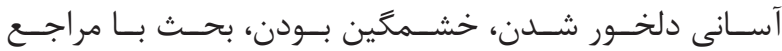

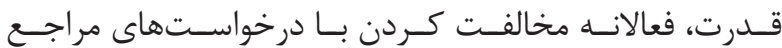

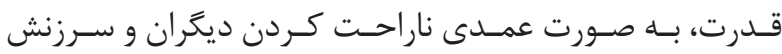

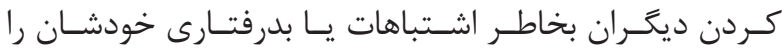

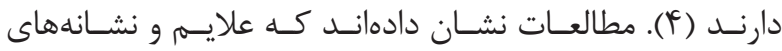

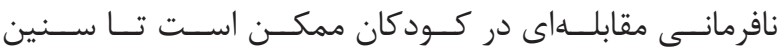

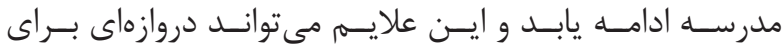

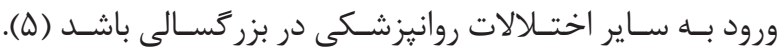

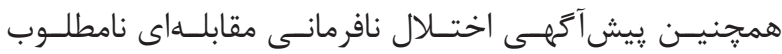

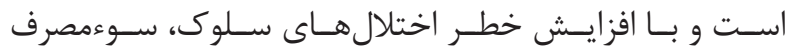

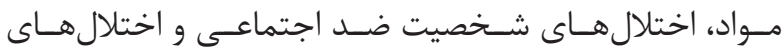

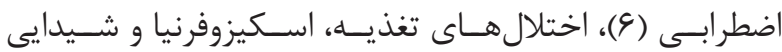
(V)

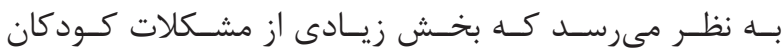

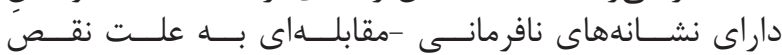

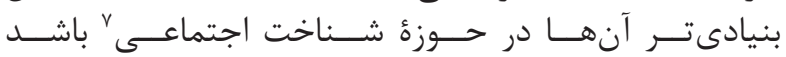
(N-IF)

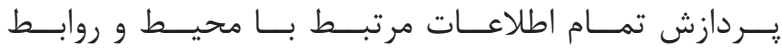

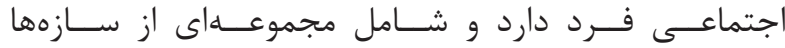

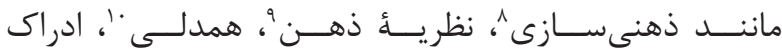

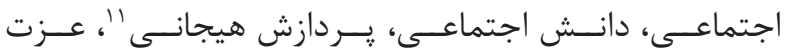

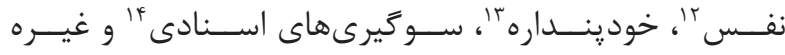

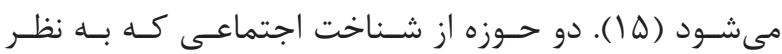

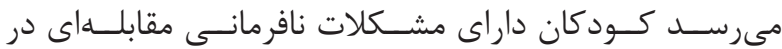

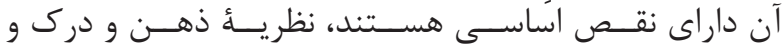

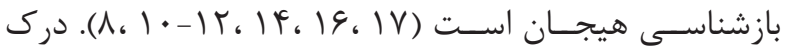

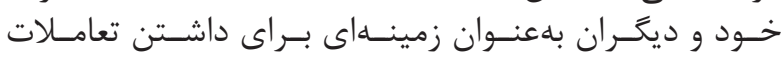

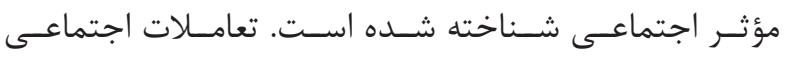

\footnotetext{
${ }^{8}$ Mentalizing

${ }^{9}$ Theory of mind

${ }^{10}$ Empathy

${ }^{11}$ Emotion processing

${ }^{12}$ Self-esteem

${ }^{13}$ Self-concept

${ }^{14}$ Attributional biases
} 


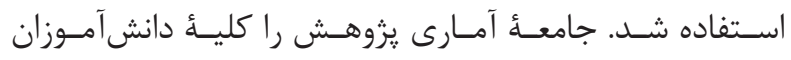

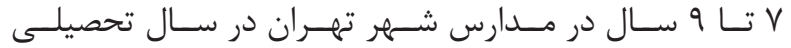

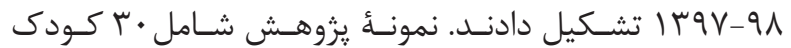

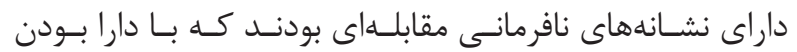

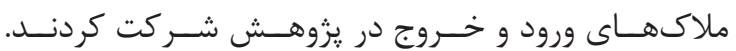

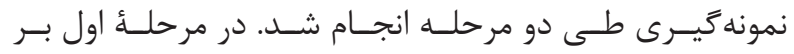

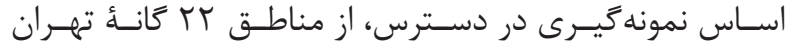

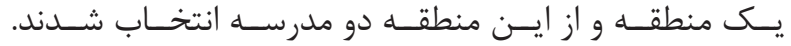

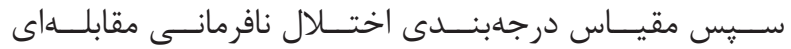

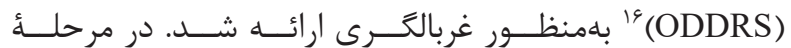

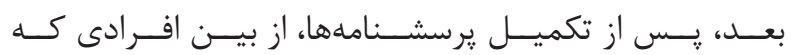

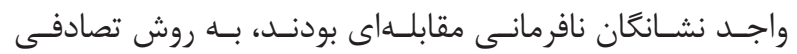

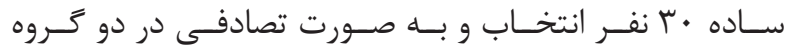

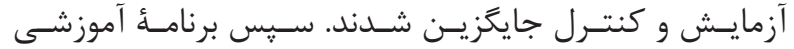

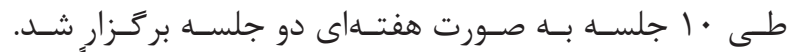

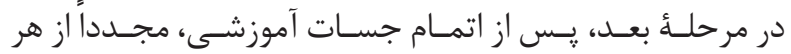

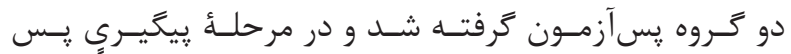

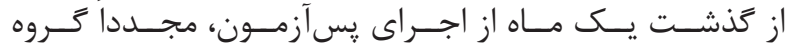

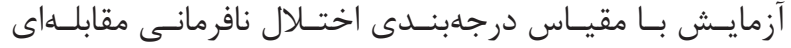

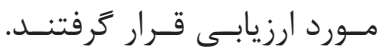

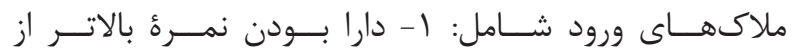

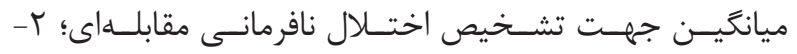

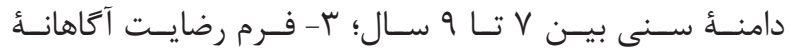

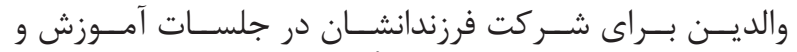

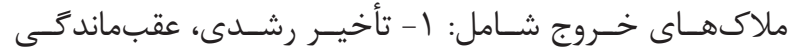

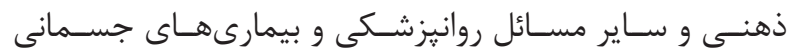

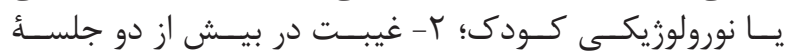

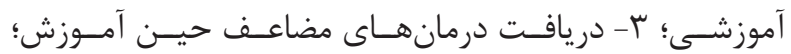

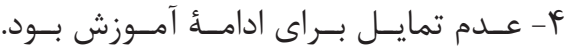

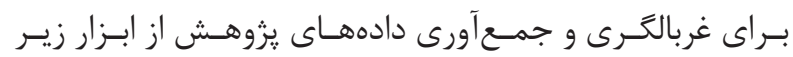

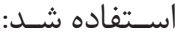

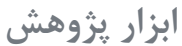
مقياس درجهبندى اختلال نافرمانى مقابلهاى

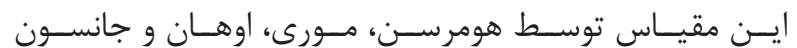

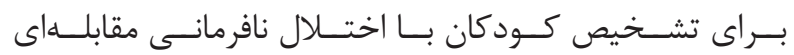

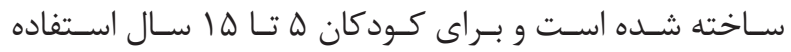

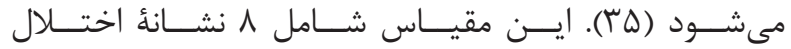

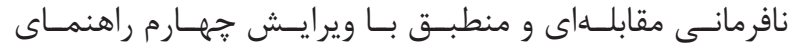

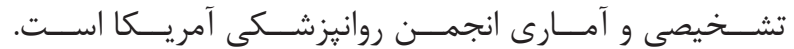

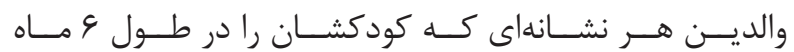

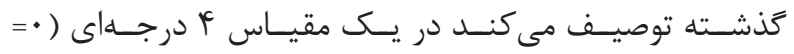

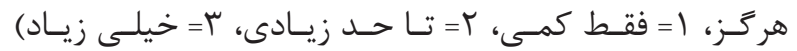

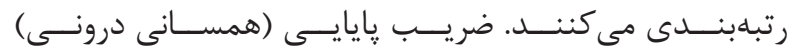

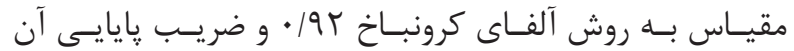

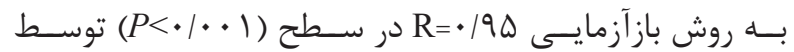

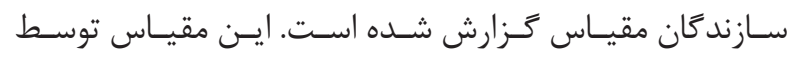

${ }^{15}$ Jacobs

${ }^{16}$ Oppositional defiant disorder rating scale

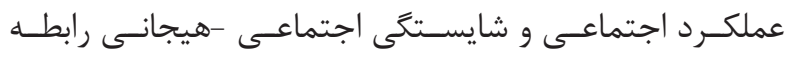

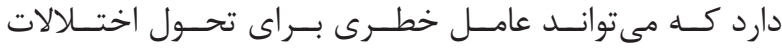

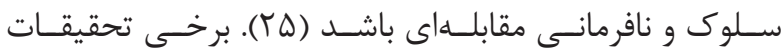

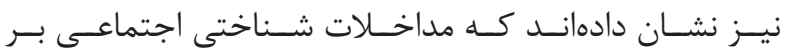

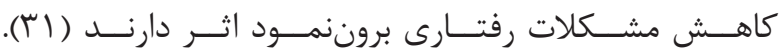

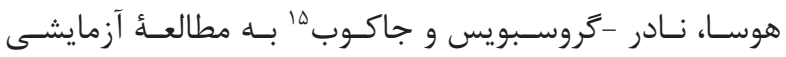

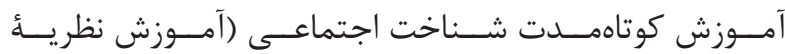

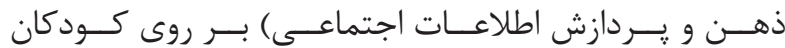

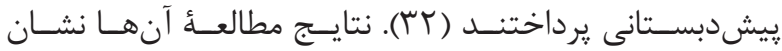

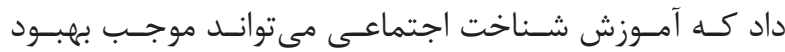

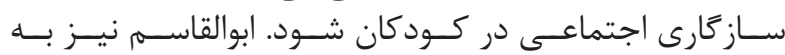

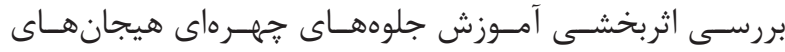

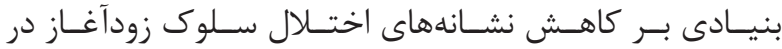

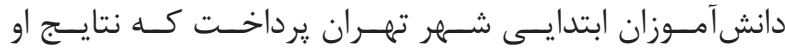

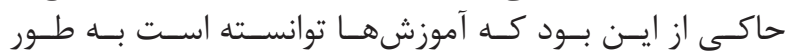

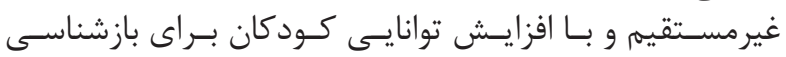

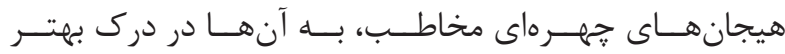

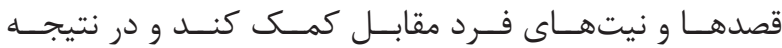

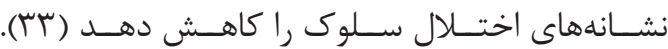

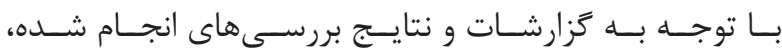

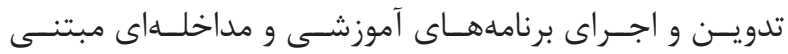

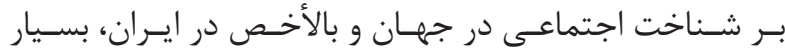

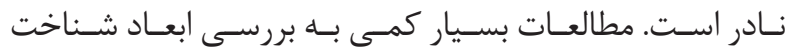

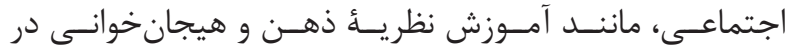

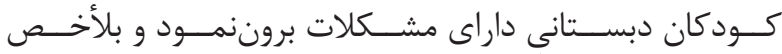

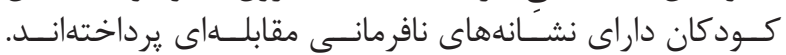

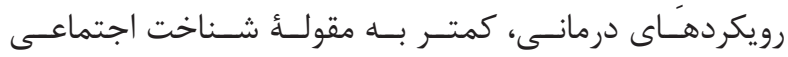

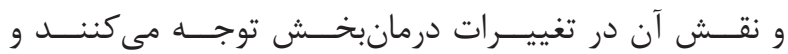

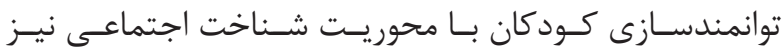

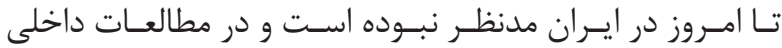

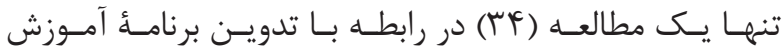

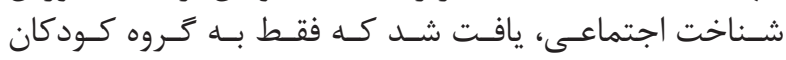

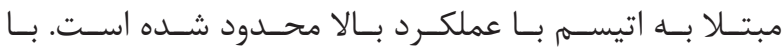

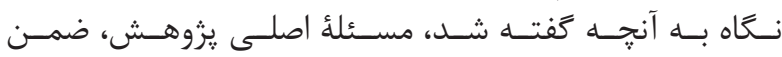

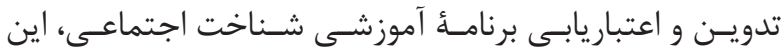

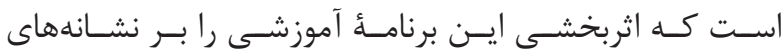

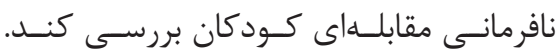

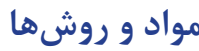

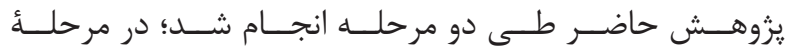

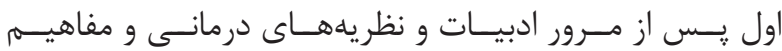

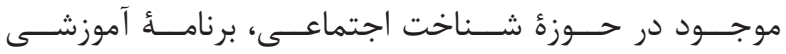

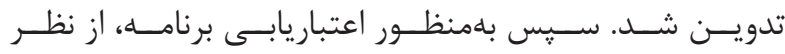

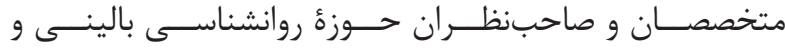

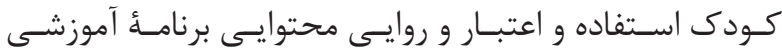

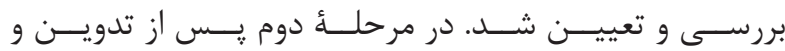

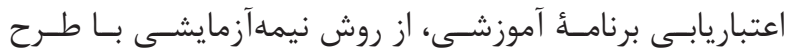

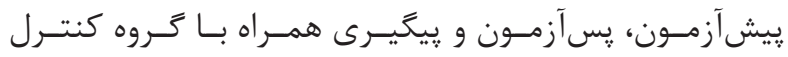




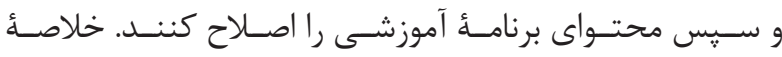

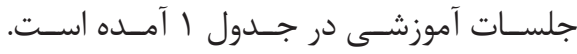

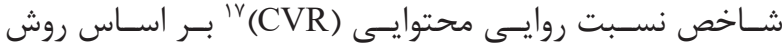

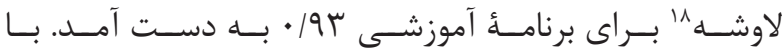

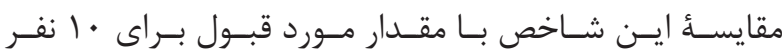

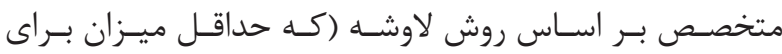

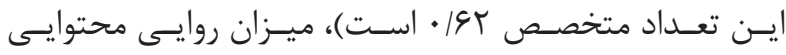

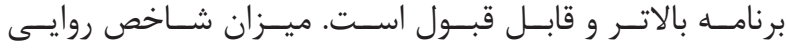

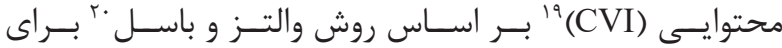

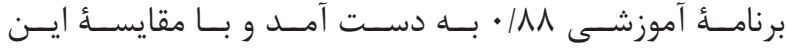

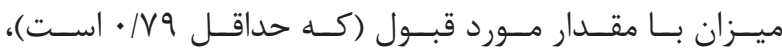

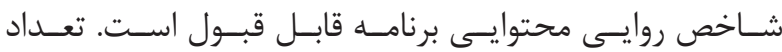

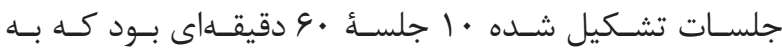

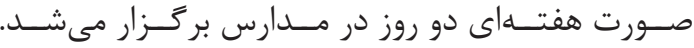

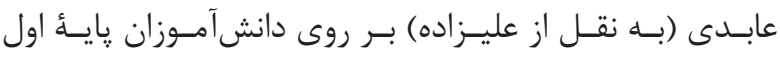

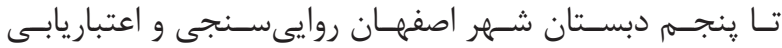

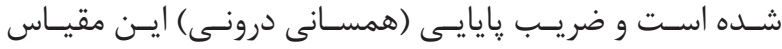

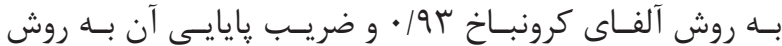

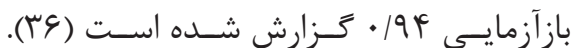

\section{برنامه آموزشى شناخت اجتماعى}

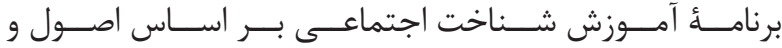

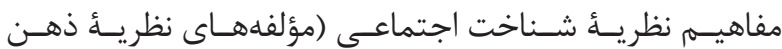

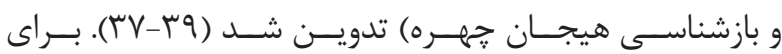

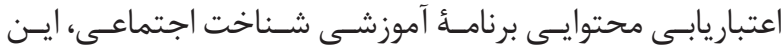

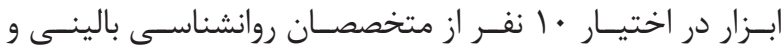

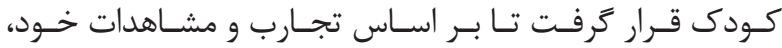

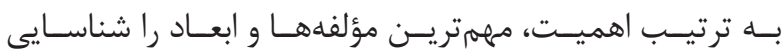

\section{مجتواي جلسه}

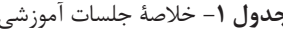

\begin{tabular}{|c|c|c|}
\hline محتواي بلسيه & عنوان جلسيه & جلميك \\
\hline 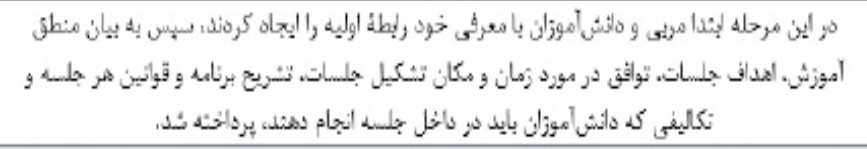 & 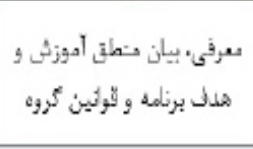 & $J_{9}$ \\
\hline 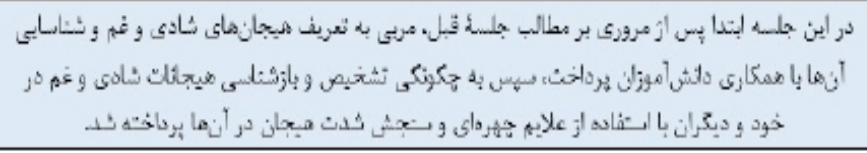 & أموزش تشخيت و بازثناسىى & دo \\
\hline 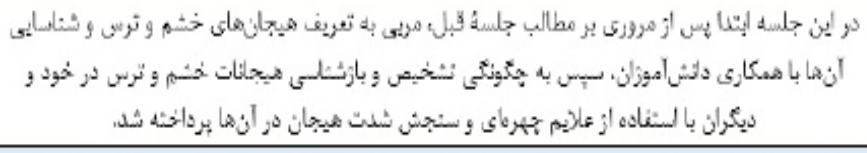 & 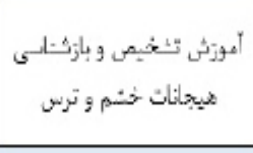 & سوت \\
\hline 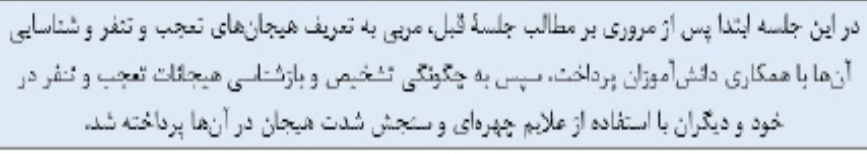 & 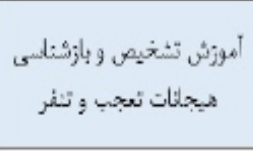 & ج جارم \\
\hline 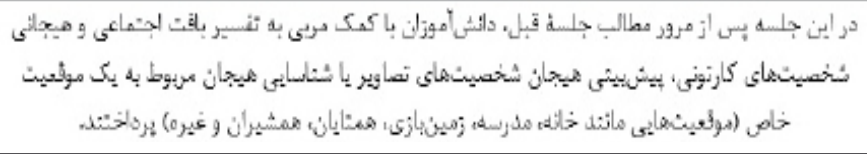 & 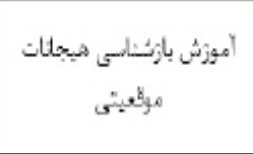 & A: \\
\hline 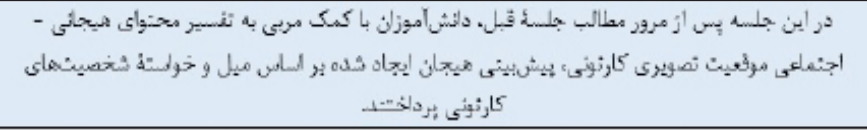 & اموزش بازتشناسى هيجـاتات & $a^{*}$ \\
\hline 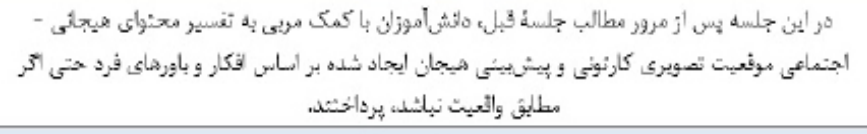 & 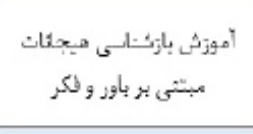 & هفتم \\
\hline 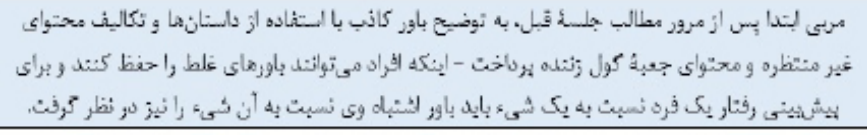 & آموزت مرك باور غلط & ot \\
\hline 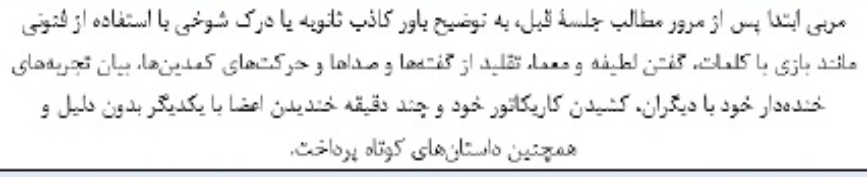 & 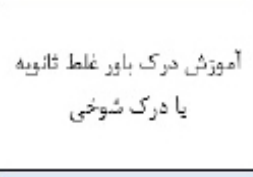 & ' \\
\hline 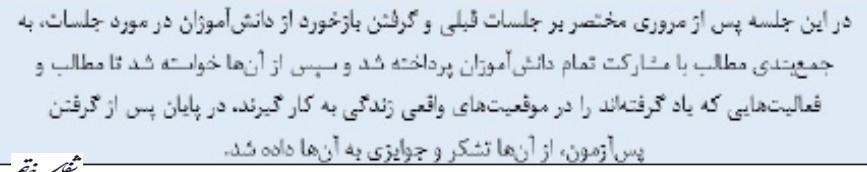 & 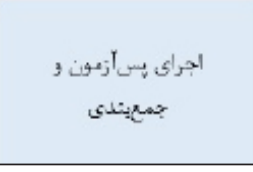 & مs \\
\hline
\end{tabular}

${ }^{17}$ Content validity ratio

${ }^{18}$ Lawshe
${ }^{19}$ Content validity index

${ }^{20}$ Waltz and Bausell 


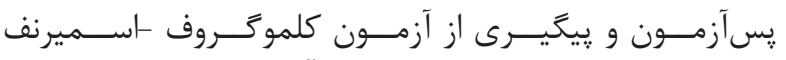

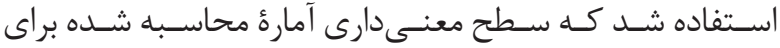

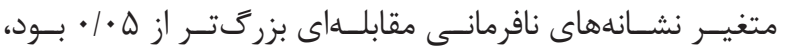

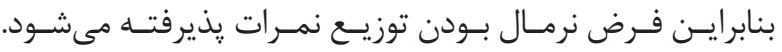

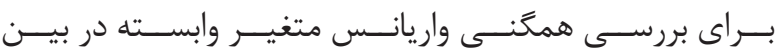

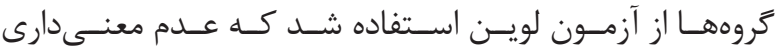

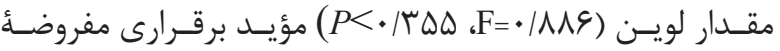

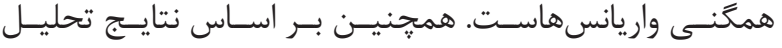

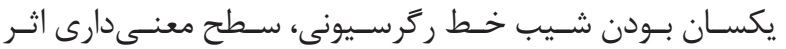

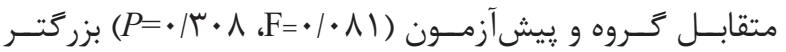

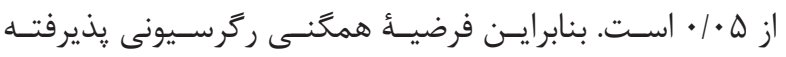

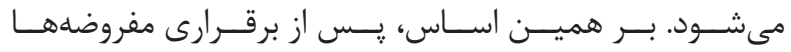

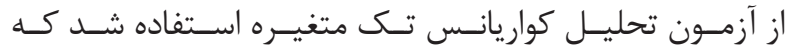

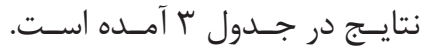

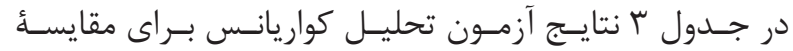

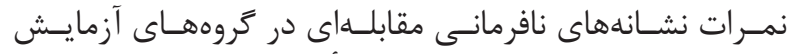

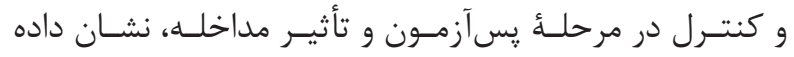

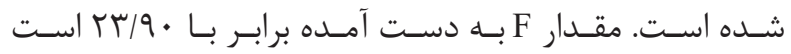

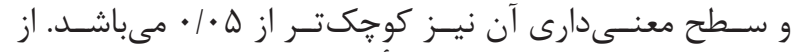

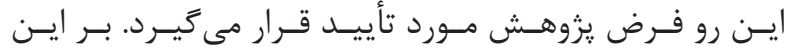

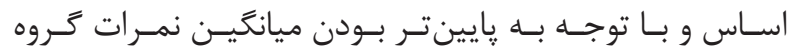

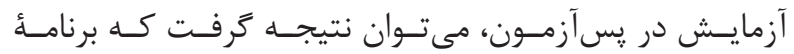

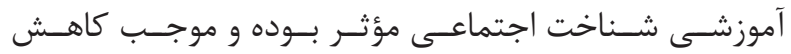

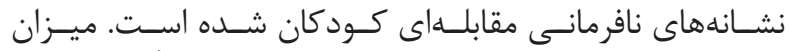

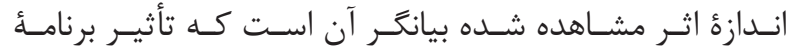
آموزشـى در حـد متوســ بـوده اسـت.

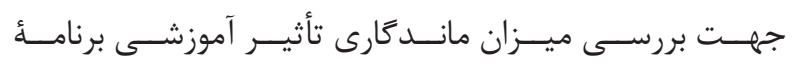

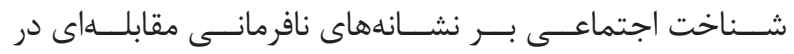

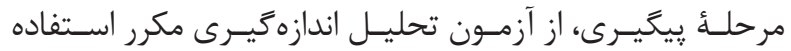

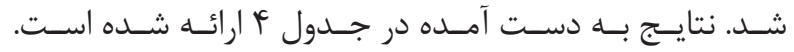

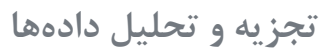

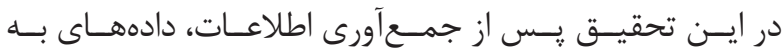

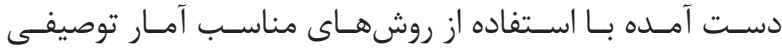

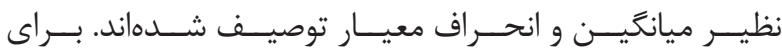

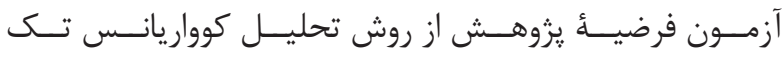

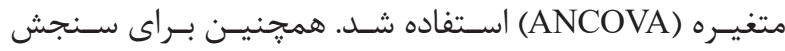

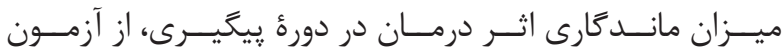

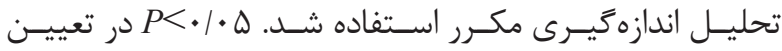

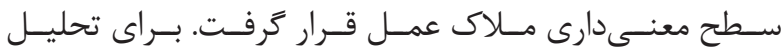

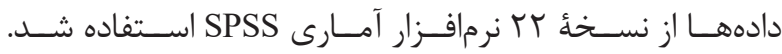

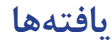

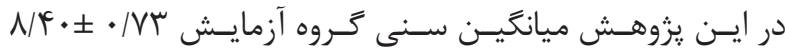

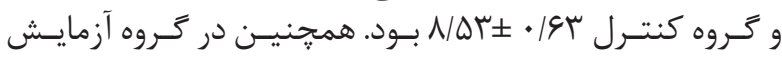

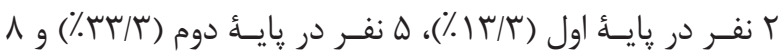

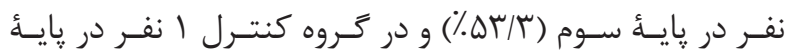

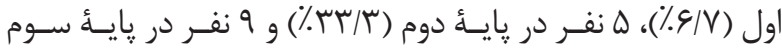

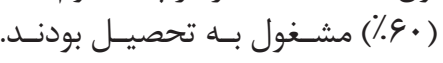

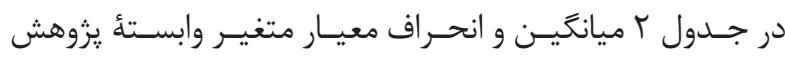

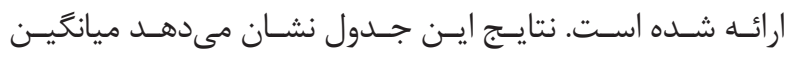

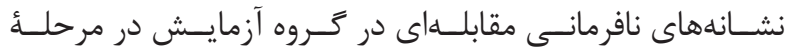

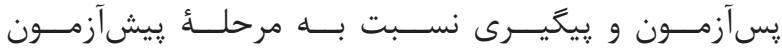

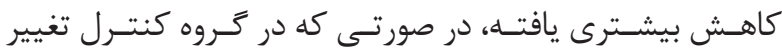

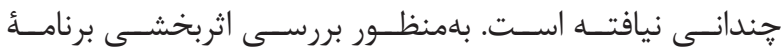

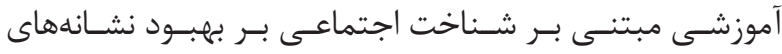

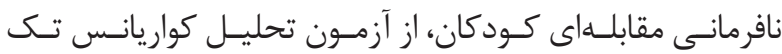

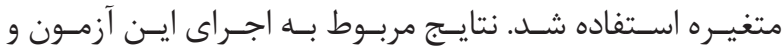

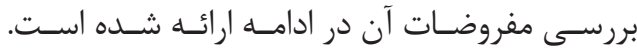

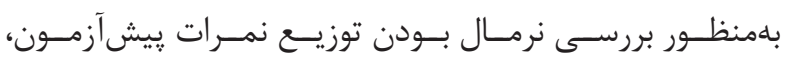

\begin{tabular}{|c|c|c|c|c|c|c|c|}
\hline \multicolumn{2}{|c|}{ ييكيرى } & \multicolumn{2}{|c|}{ به } & \multicolumn{2}{|c|}{ يِيشآزمون } & \multirow[b]{2}{*}{ 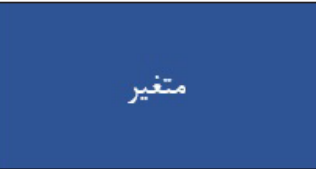 } & \multirow[b]{2}{*}{ كروه } \\
\hline أنحراف & ميانكين & أنحراف أندارد & ميانكين & أستاندارد & ميانكين & & \\
\hline $1 / 19$ & $1 r / 9 \pi$ & $r / r)$ & $11 / r$. & $1 / \wedge \Lambda$ & $14 / 9$. & نشانههاى نافرمانى مقابلهاى & آزمايش \\
\hline & - & T/FF & $14 / 19$ & r/rq & $1 \Delta / \Delta T$ & نشانههاى نافرمانى مقابلهاى & كنترل \\
\hline
\end{tabular}

جدول بـ نتايج تحليل كواريانس جهت مقايسٔ نشانهاى نافرمانى مقابلهاى در كروه آزمايش و كنترل.

\begin{tabular}{|c|c|c|c|c|c|c|}
\hline اندازئ اثر & سطح معنى دارى & Fقدار F مق F & ميانكَين مجذورات & درجئ آزادى & مجموع مجذورات & منبع تغييرات \\
\hline$\cdot / \Delta \Delta V$ & $\cdot / \cdots 1$ & $r \mu / \cdots r$ & 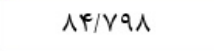 & 1 & 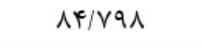 & هيشآزمون \\
\hline$\cdot / F V$. & $\cdot / \cdots 1$ & $r r / q .$. & $\Delta q / \varepsilon \cdot T$ & 1 & $\Delta q / \varepsilon \cdot r$ & كروه \\
\hline - & - & - & r/fqf & tr & GV/TrL & خطا \\
\hline- & - & - & - & rq & $r \Delta T / Q \& V$ & كل \\
\hline
\end{tabular}

\begin{tabular}{|c|c|c|c|c|}
\hline سطح معنىدارى & درجة آزادى & مقدار خىدو & آماره مخلى & متغير \\
\hline$\cdot / 1 / f$ & r & f/rfe & $\cdot / V I S$ & نشانه هاى نافر مانى مقابلهاى \\
\hline
\end{tabular}




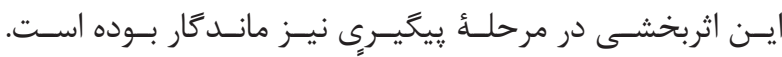

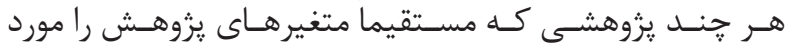

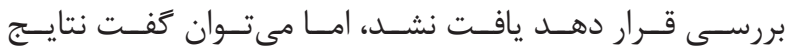

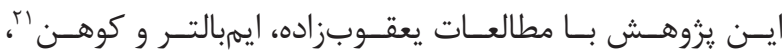

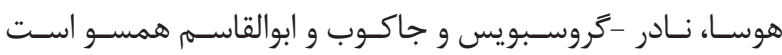

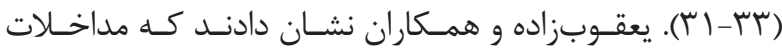

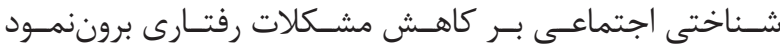

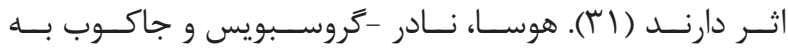

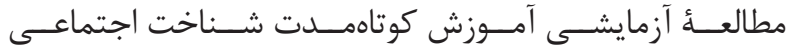

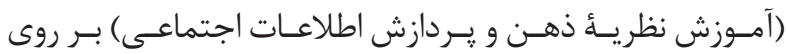

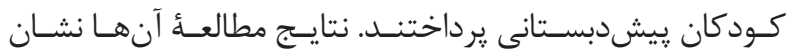

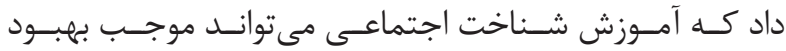

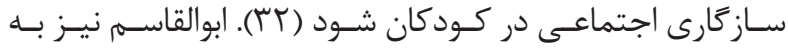

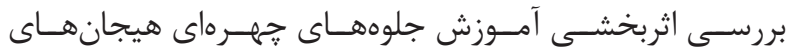

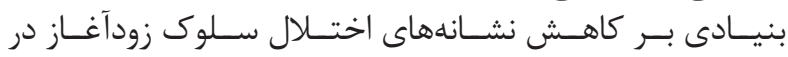

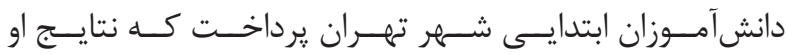

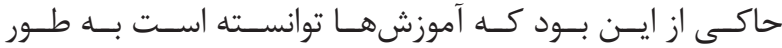

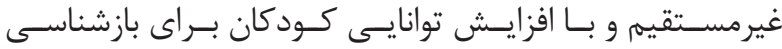

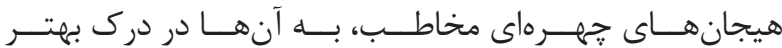

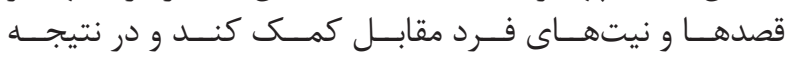

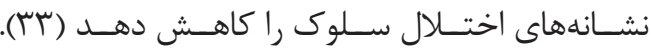

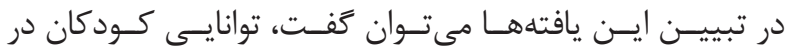

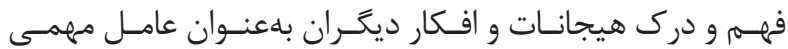

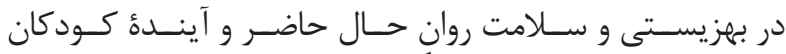

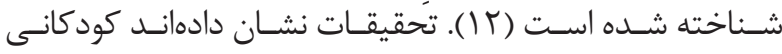

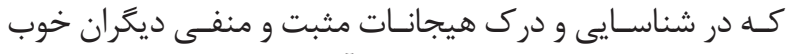

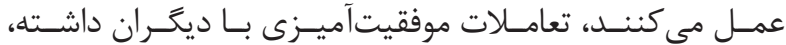

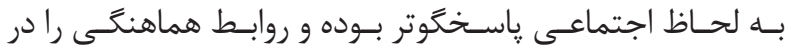

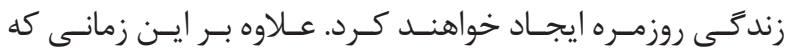

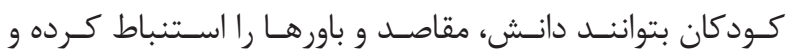

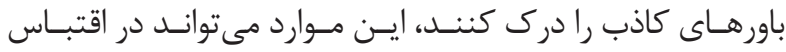

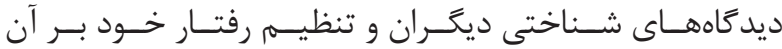

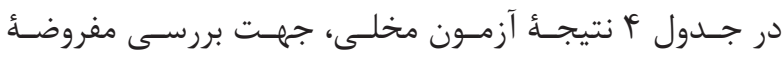

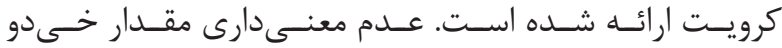

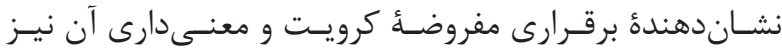

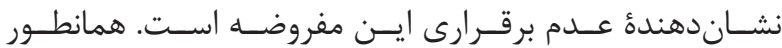

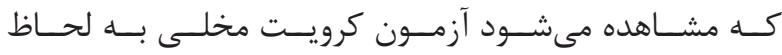

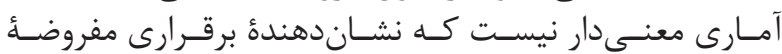
كرويست اسـت معنت

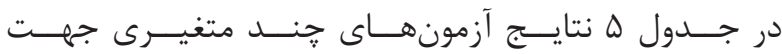

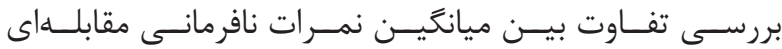

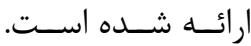

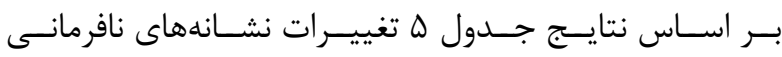

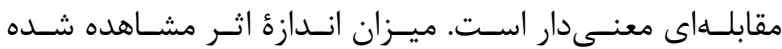

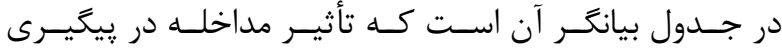

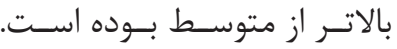

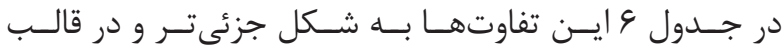

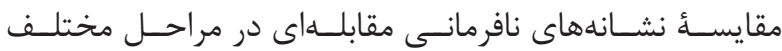

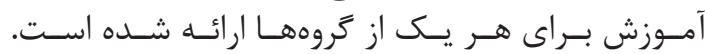

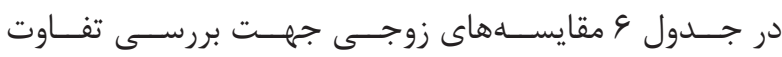

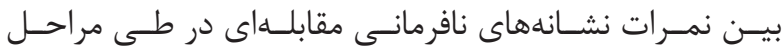

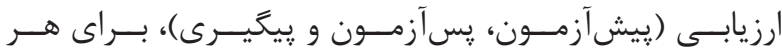

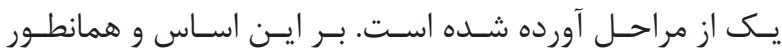

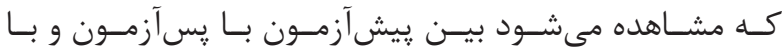

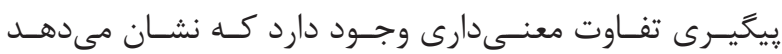

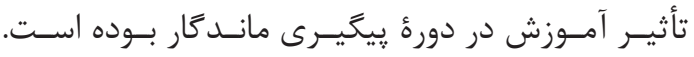

\section{بحث و نتيجه كيرى}

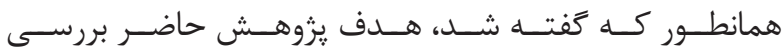

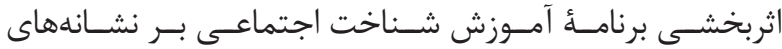

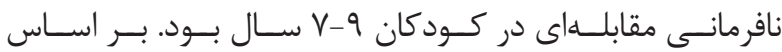

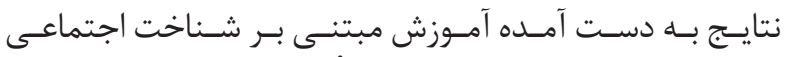

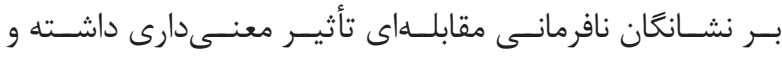

جدول ها - نتايج آزمون جند متغيرى براى بررسى ميزان ماندكًارى آموزش بر نشانهها در دوره يِيَيرى.

\begin{tabular}{|c|c|c|c|c|c|c|}
\hline اندازهة اثر & سطح معنى دارى & درجهُ آزادى خطا & درجهُ آزادى اثر & $\mathbf{F}$ & مقادير & آزمون \\
\hline$\cdot / \lambda \cdot T$ & $\cdot / \cdots 1$ & ir & r & $r E / T M$ & $\cdot / \lambda \cdot r$ & اثر ييلايى \\
\hline$\cdot / \lambda \cdot T$ & $\cdot / \cdots 1$ & ir & $r$ & YG/TM & $\cdot / 191$ & لامبداي ويلكز \\
\hline$\cdot / \Lambda \cdot T$ & $\cdot|\cdots|$ & ir & $r$ & TE/TM & $f / \cdot \varepsilon$. & اثر هتلينك \\
\hline$\cdot / \Lambda \cdot r$ &. $\mid \cdots 1$ & ir & $r$ & RE/TMA & $\psi / .9$. & بزركترين ريشهروى \\
\hline
\end{tabular}

جدول 9- نتايج مقايسهاى زوجى نشانهاى نافرمانى مقابلهاى كروه آزمايش در مراحل مختلف (ييش آزمون، يس آزمون و پييكيرى).

\begin{tabular}{|c|c|c|c|c|c|}
\hline سطح معنى دارى & خطاى استاندارد & تفاوت ميانكين دو مر حله & مرحلة (j) & مرحلة (i) & متغير \\
\hline$\cdot / \cdot 1$ & $\cdot / \Delta \cdot \Delta$ & $r / \varphi$. & بـ بس آزمون & \multirow{2}{*}{ يَيش آزمون } & \multirow{3}{*}{ نشاته هاى نافرمانى } \\
\hline$\cdot 1 \cdot \cdot 1$ & $\cdot / r \cdot r$ & 1/99 & يّيكيرى & & \\
\hline$\because \cdot r$ & - & $-1 / V r$ & يَيكيرى & لِس آزمون & \\
\hline
\end{tabular}

${ }^{21}$ Yaghoub Zadeh, Im-Bolter, and Cohen 


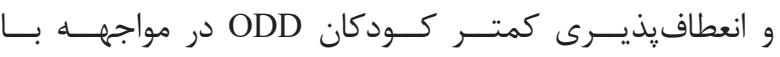

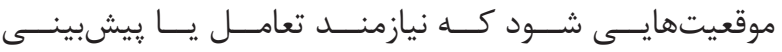

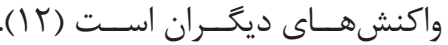

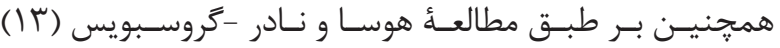

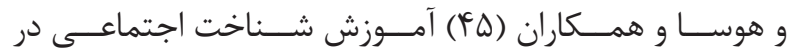

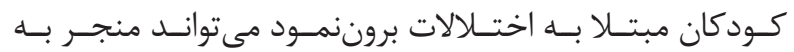

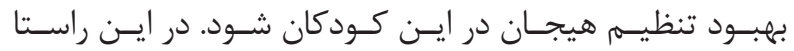

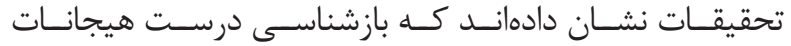

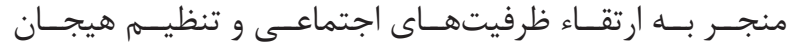

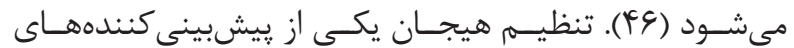

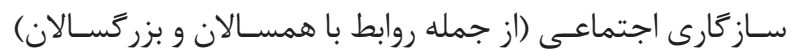

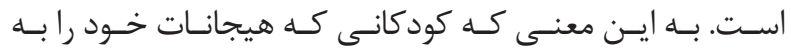

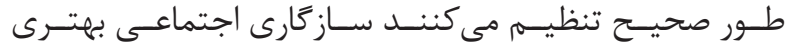

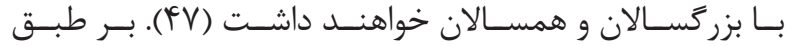

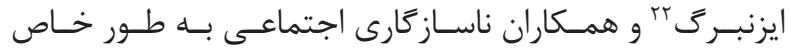

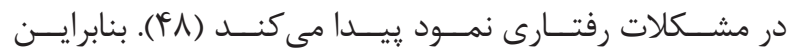

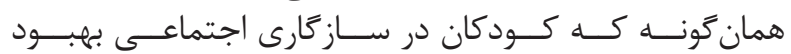

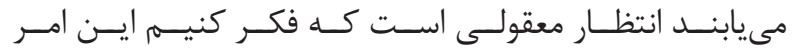

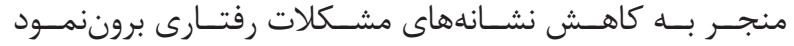

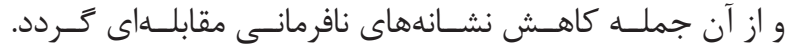

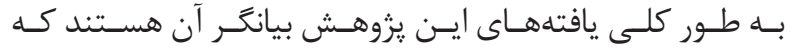

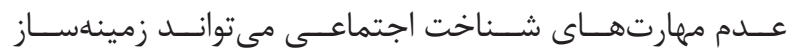

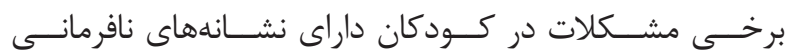

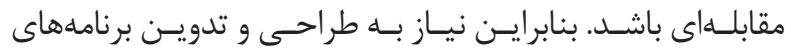

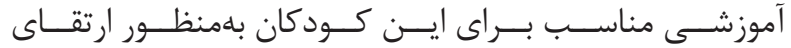

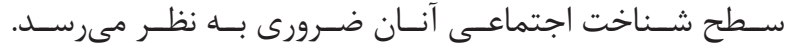

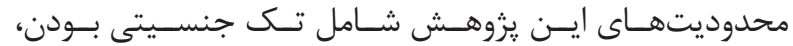

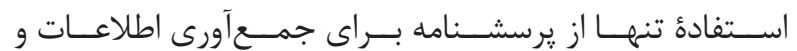

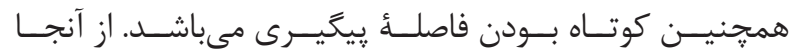

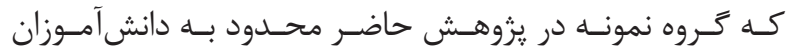

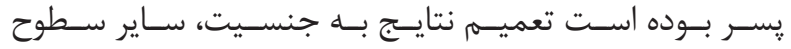

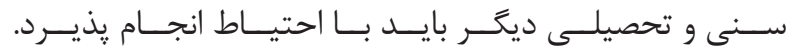

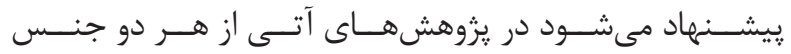

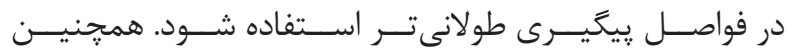

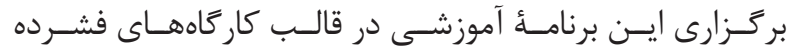

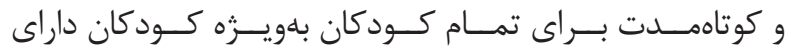

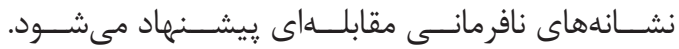

$$
\text { تشكر و قدردانى }
$$

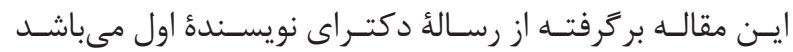

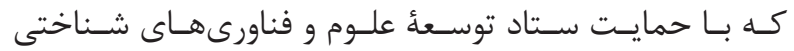

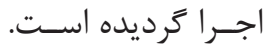

1. Keenan K. Mind the gap: assessing impairment among children affected by proposed revision to the diagnostic criteria for oppositional defiant disorder. J Abnorm Psychol. 2012; 121(3): 352-9.

2. Wilcox G, Heudes A. Clinical reasoning in the

${ }^{22}$ Eisenberg

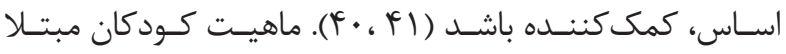

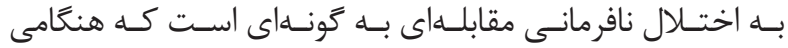

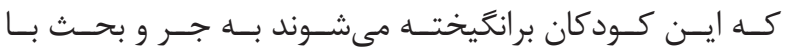

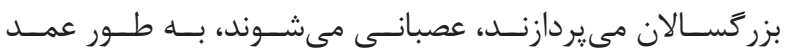

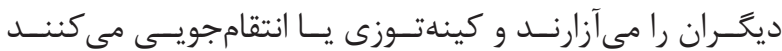

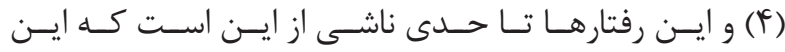

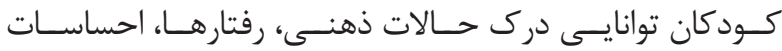

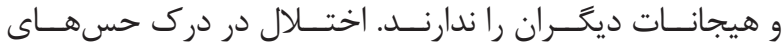

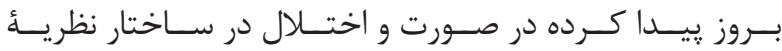

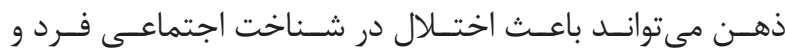

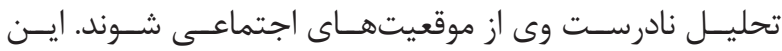

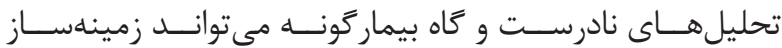

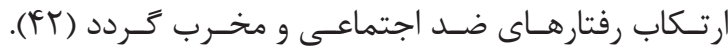

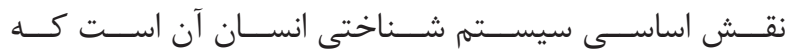

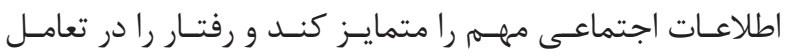

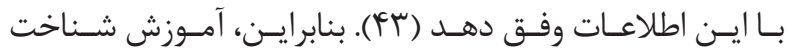

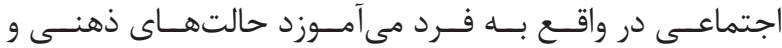

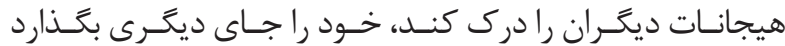

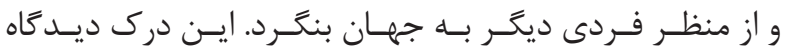

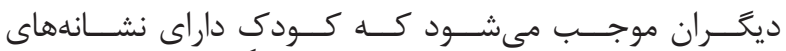

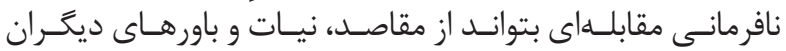

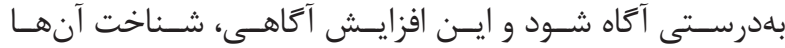

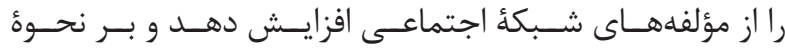

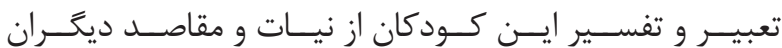

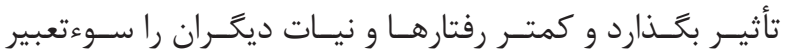

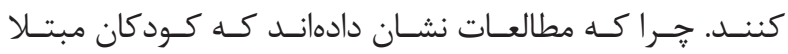

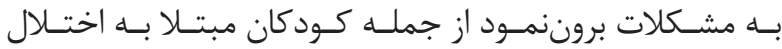

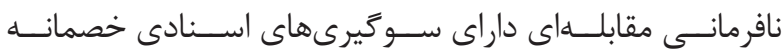

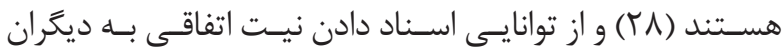

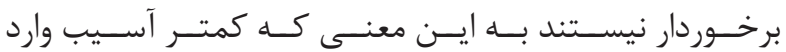

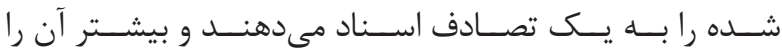

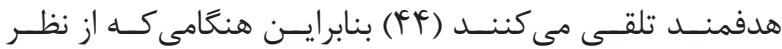

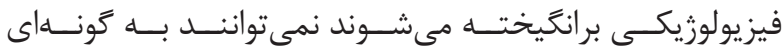

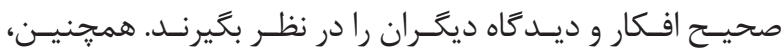

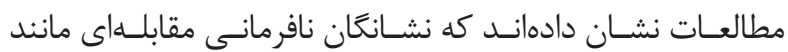

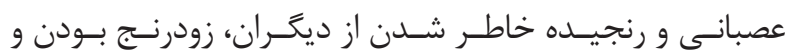

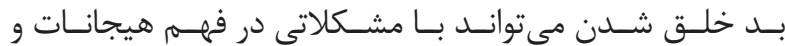

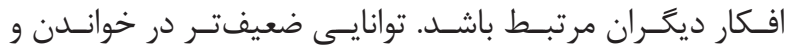

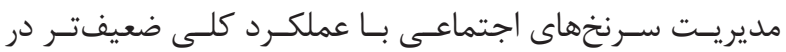

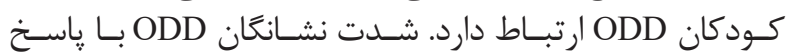

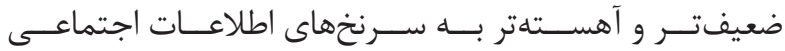

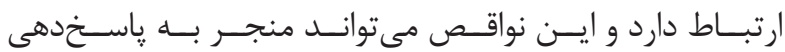

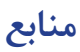
assessment and planning for intervention for oppositional defiant disorder. Can J Sch Psychol. 2017; 32(1): 46-58.

3. Sohrabi F, Asadzadeh H, Arabzadeh Z. Effectiveness of Barkley's parental education program in reducing the symptoms of oppositional defiant disorder on preschool 
children. J Sch Psychol. 2014; 3(2): 82-103.

4. American psychiatry association, text revision. diagnostic and statistical manual of mental disorders. $5^{\text {th }}$ ed. Washington, DC: Author. 2013.

5. Szentivanyi D, Balazs J. Quality of life in children and adolescents with symptoms or diagnosis of conduct disorder or oppositional defiant disorder. Ment Health Prev. 2018; 10: 1-8.

6. Folino A. The effects of antecedent exercise on students' aggressive and disruptive behaviors: exploratory analysis of temporal effects and mechanism of action. $\mathrm{PhD}$ thesis. University of Toronto. Department of Human Development and Applied Psychology. 2011.

7. Kim- Cohen J, Caspi A, Moffi TT, Harrington H, Milne BJ, Poulton R. Prior juvenile diagnoses in adults with mental disorder: Developmental follow - back of a prospective - longitudinal cohort. Arch Gen Psychiatry. 2003; 60: 709-17.

8. Crick NR, Dodge KA. A review and reformulation of social information-processing mechanisms in children's social adjustment. Psychological Bulletin. 1994; 115: 74-101.

9. Coy K, Speltz M, Deklyen M, Jones K. Socialcognitive processes in preschool boys with and without oppositional defiant disorder. J Abnorm Child Psychol. 2001; 29(2): 107-19.

10. Runions KC, Keating DP. Young children 's Social Information Processing: Family antecedents and behavioral correlates. Dev Psychology. 2007; 43(4): 838-49.

11. Denham SA, Zinsser K, Bailey CS. Emotional intelligence in the first five years of life. Encyclopedia on Early Childhood Development. George Mason University. USA. 2011.

12. Osa ND, Granero R, Domenech JM, Shamay-Tsoory $\mathrm{S}$, Ezpeleta L. Cognitive and affective components of theory of mind in preschoolers with oppositional defiance disorder: clinical evidence. Psychiatry Res. 2016; 241: 128-34.

13. Houssa M, Nader-Grosbois N. Could social cognition training reduce externalizing behaviors and social maladjustment in preschoolers? J Psychol Abnorm. 2016; 1: 1-10.

14. Yazdi SM, Bani-Jamali ShS, Salemi S. The comparison of 6-8 years girl students' performance with and without oppositional defiant disorder in theory of mind tasks. J Fundament Ment Health. 2013; 15(58): 136-45.

15. Sharp C, Croudace TJ, Goodyer IM. Biased mentalising in children aged 7-11: latent class confirmation of response styles to social scenarios and associations with psychopathology. Soc Dev. 2007; 16(1): 81-202.

16. Noordermeer SD, Luman M, Buitelaar JK, Hartman CA, Hoekstra PJ, Franke B, et al. Neurocognitive deficits in ADHD disorder with and without comorbid ODD. J Atten Disord. 2015. pii: 1087054715606216.

17. Matthys W, Vanderschuren LJ, Schutter DJ, Lochman JE. Impaired neurocognitive functions affect social learning processes in oppositional defiant disorder and conduct disorder: implications for interventions. Clin Child Fam Psychol Rev. 2012; 15(3): 234-46.

18. Amin-Yazdi SA. Social cognition and language. Journal of Mashhad School of Literature and Humanities. 2007; 40(4): 115-34.

19. Blakemore SJ. The developing social brain: implications for education. Neuron. 2010; 65: 744-7.

20. Harris PL, De Rosnay M, Pons F. "Understanding emotion". Lewis F. Havilang J, Feldman Barrett M. Handbook of Emotions. $4^{\text {th }}$ ed. New York: Guilford Press. 2016; 293-306.

21. Izard C, Fine S, Schultz D, Mostow A, Ackerman B, Youngstrom E. Emotion knowledge's a predictor of social behavior and academic competence in children at risk. J Psychol Sci. 2001; 12(1): 18-23.

22. Gotter J, Granger K, Backx R, Hobbs M, Yen Looi $\mathrm{GH}$, Barnett LH. Social cognitive dysfunction as a clinical marker: A systematic review of meta-analyses across 30 clinical conditions. Neurosci Biobehav Rev. 2018; 84(2018): 92-9.

23. Winter K, Spengler S, Bermpohl F, Singer T, Kanske $P$. Social cognition in aggressive offenders: impaired empathy, but intact theory of mind. Sci Rep. 2017; 7(1): 670. doi: 10.1038/s41598-017-00745-0.

24. Hubbard JA, Dodge KA, Cillessen AHN, Coie JD, Schwartz D. The dyadic nature of social information processing in boy's reactive and proactive aggression. J Pers Soc Psychol. 2001; 80: 268-80.

25. Mand W, Skuse D, Steer STC, Pourcain B, Oliver BR. Oppositionality and socioemotional competence: interacting risk factors in the development of childhood conduct disorder symptoms. J Am Acad Child Adolesc Psychiatry. 2013; 52(7): 718-27. 
26. Oliver BR, Barker ED, Mandy WPL, Skuse DH, Maughan B. Social cognition and conduct problems: a developmental approach. J Am Acad Child Adolesc Psychiatry. 2011; 50(4): 385-94.

27. Gambin M, Gambin T, Sharp C. Social cognition, psychopathological symptoms, and family functioning in a sample of inpatient adolescents using variablecentered and person-centered approaches. J Adolesc. 2015; 45: 31-43.

28. Dodge KA, Laird R, Lochman JE, Zelli A. Conduct problems prevention research group. multidimensional latent-construct analysis of children's social information processing patterns: correlations with aggressive behavior problems. Psychol Assess. 2002; 14: 60-73.

29. Sharp C. Theory of mind and conduct problems in children: deficits in reading the 'emotions of the eyes'. Cognition Emotion. 2008; 22(6): 1149-58.

30. Sterzer P, Stadler C, Poustka F, Kleinschmidt A. A structural neural deficit in adolescents with conduct disorder and its association with lack of empathy. Neuro Image. 2007; 37(1): 335-42.

31. Yaghoub Zadeh Z, Im-Bolter N, Cohen NJ. Social cognition and externalizing psychology: An investigation of the mediating role of language. J Abnorm Child Psychol. 2007; 35: 141-52.

32. Houssa M, Nader-Grosbois, Jacobs E. Experimental study of short-term training in social cognition in preschoolers. J Educ Train Stud. 2014; 2(1): 139-54.

33. Abolqasem F. The effectiveness of training of basic emotions' facial expression on decreasing symptoms of early-onset conduct disorder. MA thesis. Allameh Tabatabaee University, Psychology Faculty. 2016.

34. Rezaei S, Afroz Gh, Hasanzade S, Hejazi E, Kharazi SK. The development of social cognition training program and its effectiveness for emotional skills (reading, interpreting, expression) of high- functional autistic children. Journal of Research in School Learning. 2014; 1(4): 77-84.

35. Hommersen P, Murray C, Ohan J, Johnston C. Oppositional defiant disorder rating scale: preliminary evidence of reliability and validity. J Emot Behav Disord. 2006; 14(2): 118-25.

36. Alizadeh M. The effectiveness of social adjustment skills training on the symptoms of oppositional defiant and theory of mind in children with oppositional defiant disorder. MA thesis. Allameh Tabatabaee University, psychology Faculty. 2016.

37. Howlin P, Baron-Cohen S, Hadwin J. Teaching children with autism to mind-read: a practical guide for teachers and parents. Chichester, NY: John Wiley \& Sons, Inc. 1999.

38. Muris P, Steerneman P, Meesters C, Merckelbach H, Horselenberg R, van den Hogen T, et al. The TOM test: A new instrument for assessing theory of mind in normal children and children with pervasive developmental disorders. J Autism Dev Disord. 1999; 29(1): 67-80.

39. Ekman P, Friesen W. "Photographs of facial affect recognition test". Consulting Psychologists Press. 1976; 221-31.

40. Nader-Grosbois N, Houssa M, Mazzone S. How could theory of mind contribute to the differentiation of social adjustment profiles of children with externalizing behavior disorders and children with intellectual disabilities? Res Dev Disabil. 2013; 34: 2642-60.

41. Deneault J, Ricard M. Are emotion and mind understanding differently linked to young children's social adjustment? relationships between behavioral consequences of emotions, false belief, and SCBE. J Genet Psychol. 2013; 1: 88-116.

42. Ekhtiari H, Safaei G, Esmaeeli-Djavid G, Ganjgahi H, Naderi P, Mokri A. Study of Social cognition impairment among opiate dependents. Soc Welf. 2011; 11(41): 399-420.

43. Grossmann T, Johnson MH. The development of the social brain in human infancy. Eur J Neurosi. 2007; 25(4): 909-19.

44. Webster-Stratton C, Lindsay DW. Social competence and conduct problems in young children: issues in assessment. J Clin Child Psychol. 1999; 28(1): 25-43.

45. Houssa M, Nader-Grosbois, Jacobs E. Impact of short-term training in social cognition in preschoolers with externalizing behavior. J Edu Train Stud. 2017; 5(1): 110-23.

46. Leung D, Ordqvist A, Falkmer T, Parsons R. Falkmer M. Facial emotion recognition and visual search strategies of children with high functioning autism and Asperger syndrome. Res Autism Spectr Disord. 2013; 7(7): 833-44.

47. Denham SA. Dealing with feelings: how children negotiate the worlds of emotions and social relationships. Cogn Brain Behav. 2007; 11: 1-48.

48. Eisenberg N, Valiente C, Spinrad TL, Cumberland A, Liew J, Reiser M, et al. Longitudinal relations of children's effortful control, impulsivity, and negative emotionality to their externalizing, internalizing, and cooccurring behavior problems. Dev Psychol. 2009; 45: 988-1008. 